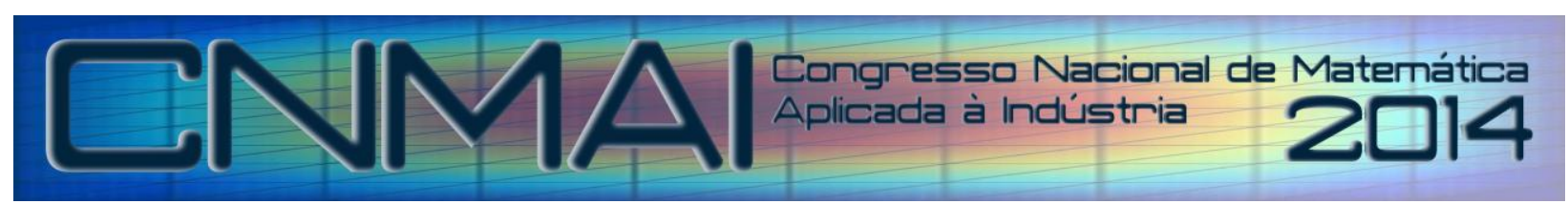

18 a 21 de novembro de 2014, Caldas Novas - Goiás

\title{
ANÁLISE ESTÁTICA LINEAR DE VIGA LAMINADA DE PVDF
}

\author{
Edgar Della Giustina, edgar.giustina@pr.senai.br ${ }^{1}$ \\ ${ }^{1}$ Faculdade de Tecnologia SENAI CIC - Departamento de Educação ... Rua Senador Accioly Filho, 298, Cidade \\ Industrial, CEP 81310-000, Curitiba, PR - Brasil
}

\begin{abstract}
Resumo: O presente trabalho visa analisar o comportamento de vigas de material compósito piezoelétrico (PVDF). A análise ocorre mediante desenvolvimento de rotinas no compilador FORTRAN para inclusão de propriedades e de matrizes constitutivas dos materiais piezoelétricos. Para a resolução utilizou-se a Teoria de Deformações Cisalhante de Primeira Ordem (FSDT). O método numérico empregado foi o Método Modificado da Função de Green Local (MMFGL). Para a obtenção de melhores resultados foram criadas malhas de diferentes dimensões até conseguir uma convergência de resultados. Os resultados obtidos foram comparados com vários métodos numéricos encontrados na literatura e com soluções analíticas de materiais piezoelétricos submetidos a tensões elétricas. Materiais piezoelétricos podem ser utilizados em estruturas que precisa de uma boa flexibilidade e de pequenos deslocamentos. Com isso, esse artigo pretende mostrar uma colaboração para melhor compreender o comportamento das características dos materiais piezoelétricos em relação aos que não apresentam tais propriedades.
\end{abstract}

Palavras-chave: Piezoeletricidade, Material Compósito, Método Modificado da Função de Green Local.

\section{INTRODUÇÃO}

Uma viga laminada têm $n$ camadas que podem variar sua espessura e o seu material. Um dos casos, é a combinação de mais de um tipo de material, essa combinação é chamada de material compósito. A motivação para usar um material compósito é que as características mecânicas do material adquirido com essa junção são melhores do que se fossem usados cada material separadamente. Um dos tipos de compósitos que têm sido usado em alguns tipos de estruturas é compósito piezoelétrico. O compósito piezoelétrico é obtido pela combinação de um polímero e de um material com propriedades piezoelétricas, o polímero vêm para melhorar as caraterísticas mecânicas da estrutura piezoelétrica. Por conseguir trabalhar mecanicamente e eletricamente, os materiais piezoelétricos são chamados de estruturas inteligentes.

A nova concepção de estruturas inteligentes tem sido assunto de projetos estruturais por seu poder na resolução de questões estruturais. Por relacionar as propriedades mecânicas e elétricas, materiais piezoelétricos são usados como atuadores ou sensores em estruturas inteligentes.

Esse trabalho visa analisar laminados de materiais compostos submetidos a deformações causadas por efeitos piezoelétricos. O efeito piezoelétrico é um assunto de relevância em algumas áreas que estudam melhoria de estruturas. Considerando que as configurações das aeronaves podem ser alteradas por deformações causadas por atuadores piezoelétricos que podem, ainda, ser utilizados na construção de robôs. Para aplicação do material piezoelétrico são interessantes estudos dos princípios teóricos e desenvolvimento de programas com métodos numéricos computacionais.

Na maioria dos trabalhos encontrados na literatura são feitas análises dinâmicas. No caso desse trabalho, é efetuada uma análise estática usando a Teoria da Deformação Cisalhante de Primeira Ordem e a soluções numéricas obtidas pelo Método Modificado da Função de Green Local.

\section{EQUAÇÕES CONTITUTIVAS DA PIEZOELETRICIDADE}

De acordo com Godoy (2008), o deslocamento dielétrico tem relação com o campo elétrico à deformação constante mostrada nas Eq. (1) e (2):

$$
\{D\}=[P]\{E\}
$$




$$
\{E\}=[P]^{-1}\{D\}
$$

A tensão mecânica tem relação com a deformação para campo elétrico constante conforme a Eq. (3):

$$
\{\sigma\}=\left[c^{E}\right]\{\varepsilon\}
$$

Onde:

$c^{E}\left(\mathrm{~N} / \mathrm{m}^{2}\right)$ é a matriz de rigidez elástica para campo elétrico constante;

$\sigma\left(\mathrm{N} / \mathrm{m}^{2}\right)$ é a tensão mecânica.

Relacionando as propriedades elétricas e mecânicas para o material piezelétrico, obtém as Eq. (4) e (5):

$$
\begin{gathered}
\{\sigma\}=\left[c^{E}\right]\{\varepsilon\}-[e]^{T}\{E\} \\
\{D\}=[e]\{\varepsilon\}+[P]\{E\}
\end{gathered}
$$

Onde:

$e\left(\mathrm{C} / \mathrm{m}^{2}\right)$ é a matriz de constantes piezelétricas;

As variáveis mecânicas são a deformação $\varepsilon$ e a tensão mecânica $\sigma$ e as variáveis elétricas são o deslocamento elétrico $D$ e o campo elétrico $E$. Considerando o modelo unidimensional do solido piezelétrico, tem-se aa Eq. (6) e (7):

$$
\begin{aligned}
& \{\sigma\}=\{\sigma(\varepsilon, E)\} \\
& \{D\}=\{D(\varepsilon, E)\}
\end{aligned}
$$

Ainda é possível escrever essas equações, da seguinte forma:

$$
\begin{aligned}
& \{\sigma\}=\left[c^{E}\right]\{\varepsilon\}-[e]\{E\} \\
& \{D\}=[e]\{\varepsilon\}-[P]\{E\}
\end{aligned}
$$

Os vetores do deslocamento elétrico e campo elétrico são dados nas três direções (x, y, z) pelas Eq. (10) e (11):

$$
\begin{aligned}
& D=\left\{\begin{array}{l}
D_{x} \\
D_{y} \\
D_{z}
\end{array}\right\} \\
& E=\left\{\begin{array}{l}
E_{x} \\
E_{y} \\
E_{z}
\end{array}\right\}
\end{aligned}
$$

$\mathrm{Na}$ forma matricial as equações piezoelétricas são definidas conforme a Eq. (12):

$$
\left\{\frac{\sigma}{D}\right\}=\left[\begin{array}{cc}
{\left[c^{E}\right]} & {[e]^{t}} \\
{[e]} & {[P]}
\end{array}\right]\left\{\frac{\varepsilon}{E}\right\}
$$

Pode-se escrever também na maneira da Eq. (13):

$$
\left\{\frac{\varepsilon}{D}\right\}=\left[\begin{array}{cc}
{\left[c^{E}\right]} & {[d]{ }^{t}} \\
{[d]} & {[P]}
\end{array}\right]\left\{\frac{\sigma}{E}\right\}
$$

Pela teoria da elasticidade, a matriz de rigidez elástica é dada pela Eq. (14):

$$
\left[c^{E}\right]=\left[\begin{array}{cccccr}
c_{11} & c_{12} & c_{13} & 0 & 0 & 0 \\
c_{21} & c_{22} & c_{23} & 0 & 0 & 0 \\
c_{31} & c_{32} & c_{33} & 0 & 0 & 0 \\
0 & 0 & 0 & c_{44} & 0 & 0 \\
0 & 0 & 0 & 0 & c_{55} & 0 \\
0 & 0 & 0 & 0 & 0 & c_{66}
\end{array}\right]
$$


O tensor piezoelétrico é mostrado na Eq. (15):

$$
[P]=\left[\begin{array}{ccc}
P_{11} & 0 & 0 \\
0 & P_{22} & 0 \\
0 & 0 & P_{33}
\end{array}\right]
$$

As constantes piezoelétricas que provocam tensão mecânica são distribuídas matricialmente conforme a Eq. (16):

$$
[e]=\left[\begin{array}{cccccc}
0 & 0 & 0 & 0 & e_{15} & 0 \\
0 & 0 & 0 & e_{24} & 0 & 0 \\
e_{31} & e_{32} & e_{33} & 0 & 0 & 0
\end{array}\right]
$$

Por fim, na Eq. (17) é mostrada a equação constitutiva da piezoeletricidade:

$$
\left\{\begin{array}{l}
\sigma_{11} \\
\sigma_{22} \\
\sigma_{33} \\
\sigma_{23} \\
\sigma_{31} \\
\sigma_{12} \\
D_{1} \\
D_{2} \\
D_{3}
\end{array}\right\}=\left[\begin{array}{ccccccccc}
c_{11}^{E} & c_{12}^{E} & c_{13}^{E} & 0 & 0 & 0 & 0 & 0 & e_{19} \\
c_{21}^{E} & c_{22}^{E} & c_{23}^{E} & 0 & 0 & 0 & 0 & 0 & e_{29} \\
c_{31}^{E} & c_{32}^{E} & c_{33}^{E} & 0 & 0 & 0 & 0 & 0 & e_{39} \\
0 & 0 & 0 & c_{44}^{E} & 0 & 0 & 0 & e_{48} & 0 \\
0 & 0 & 0 & 0 & c_{55}^{E} & 0 & e_{56} & 0 & 0 \\
0 & 0 & 0 & 0 & 0 & c_{66}^{E} & 0 & 0 & 0 \\
0 & 0 & 0 & 0 & e_{75} & 0 & -P_{11} & 0 & 0 \\
0 & 0 & 0 & e_{84} & 0 & 0 & 0 & -P_{22} & 0 \\
e_{91} & e_{92} & e_{93} & 0 & 0 & 0 & 0 & 0 & -P_{33}
\end{array}\right]\left\{\begin{array}{c}
\varepsilon_{11} \\
\varepsilon_{22} \\
\varepsilon_{33} \\
\varepsilon_{23} \\
\varepsilon_{31} \\
\varepsilon_{12} \\
-E_{1} \\
-E_{2} \\
-E_{3}
\end{array}\right\}
$$

\section{TEORIAS E RELAÇÕES SOBRE PLACAS}

\subsection{Teoria sobre Placas de Camada Equivalente Única}

De acordo com Machado (1992), nas Teorias de Camada Equivalente única, a placa laminada é vista como se tratasse de uma única lâmina com um único tipo de material. Suas caracterísitcas são encontradas por integração, por toda sua espessura, a partir da características de cada lâmina. Compreende-se que cada uma dessas lâminas está sendo submetida a um estado plano de tensões e que seja elástica. A placa que resulta dessas junções é quase sempre anisotrópica.

A Fig. 1 ilusta essa Teoria.

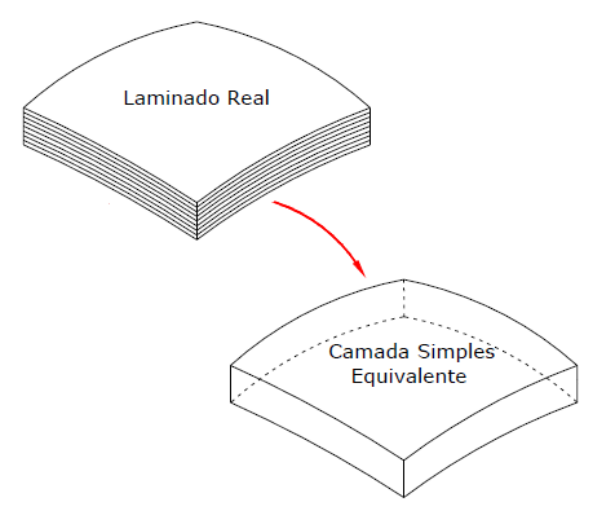

Figura 1. Teoria da Camada Equivalente Única para uma viga laminada formada por sete camadas (Faria, 2006).

\subsection{Teoria da Deformação Cisalhante de Primeira Ordem (FSDT)}

A FSDT descreve o campo de deslocamentos por meio de polinômios lineares ao longo da espessura e inclui as contribuições dos efeitos cisalhantes na análise. Em compostos, os valores dos coeficientes de correção do cisalhamento dependem da distribuição das deformações cisalhantes transversais ao longo da espessura. A precisão da FSDT diminui quando a relação espessura/comprimento da placa e o ângulo de orientações das fibras crescem (Machado, 1992).

A Fig. 2 ilustra essa Teoria. 


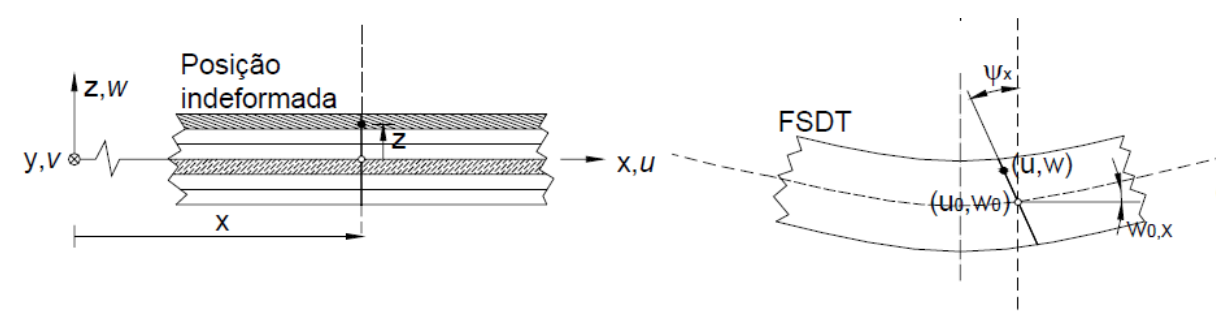

Figura 2. Representação esquemática de uma placa composta (à esquerda) e ilustração cinemática da deformação representada como uma camada equivalente única na FSDT (à direita) (Faria, 2006).

\subsection{Método Modificado da Função de Green Local (MMFGL)}

O MMFGL é um método de integral que gera um resultado ideal para o problema, sua aplicação a placas laminadas é uma opção ao Método dos Elementos Finitos. Esse método não tem fácil discretização, tem grande precisão de resultados e ótima taxa de convergência para fluxos de contorno, trata-se da união do Método do Elementos Finitos e de Elementos de Contorno (Della Giustina, (2013)).

De acordo com os artigos de Machado et al. (2013) e Machado et al. (2007), o MMFGL usa dois problemas, o real e o auxiliar. O problema real é regulado pelos operadores diferenciais $L, D$ e $N$. O problema auxiliar é regulado pelos operadores diferenciais adjuntos $L^{*} e N^{*}$.

No problema real, $u(P)$ é a variável desconhecida, $(P)$ é o termo independente, $G(P, Q)$ é a matriz de função de Green. No problema auxiliar, $\delta(P, Q)$ é a função generalizada, $I$ é o operador de identidade e $N^{\#}$ é um operador adicional. Os pontos $P$ e $Q$ são os pontos de domínio e os pontos $p$ e $q$ são os pontos de contorno. A Tab. 1 mostra o problema real e o auxiliar.

Tabela 1. Problema real e auxiliar (Machado et al., 2013).

\begin{tabular}{c}
\hline Problema real \\
\hline Encontrar u $(\mathrm{P})$ tal que \\
L $\mathbf{u}(\mathbf{P})=\mathbf{a}(\mathbf{P}), \Pi$ i $\Omega$ \\
Submetido a \\
D $\mathbf{u}(\mathrm{p})=\mathbf{b}(\mathrm{p}) ; \mathrm{p} \in \Gamma_{\mathrm{D}}$ \\
$\mathbf{N} \mathbf{u}(\mathrm{p})=\mathbf{C}(\mathrm{p}) ; \mathrm{p} \in \Gamma_{\mathrm{N}}$ \\
\hline
\end{tabular}

\begin{tabular}{|c|c|}
\hline \multicolumn{2}{|c|}{ Problema auxiliar } \\
\hline Encontrar $\mathbf{G}(\mathbf{P}, \mathbf{Q})$, de t & lodo que \\
\hline $\begin{array}{r}\mathrm{L}^{*} \mathbf{G}(\mathbf{P}, \mathbf{Q})=\boldsymbol{\delta}(\mathbf{P}, \mathbf{Q}) \mathbf{I} ; \\
\text { Submetido a } \\
\left(\mathbf{N}+\mathbf{N}^{* \#)} \mathbf{G}(\mathrm{p},\right.\end{array}$ & $\begin{array}{l}\mathrm{P}, \mathrm{Q} \in \Omega \\
0\end{array}$ \\
\hline
\end{tabular}

O tratamento direto do operador Newmann pode causar erros numéricos. É introduzido um vetor $f(p)$ que trabalha com os fluxos de contorno, e é dado pela Eq. (18):

$$
f(p)=\left(N+N^{* \#}\right) u(p)
$$

Pode-se dizer que:

$$
\begin{aligned}
& u(Q)=\int_{\Omega}\left[G^{T}(P, Q) a(P)\right] d \Omega+\int_{\Gamma}\left[G^{T}(p, Q) f(p)\right] d \Gamma ; P, Q \in \Omega ; p, \mathrm{q} \in \Gamma \\
& u(q)=\int_{\Omega}\left[G^{T}(P, Q) a(P)\right] d \Omega+\int_{\Gamma}\left[G^{T}(p, q) f(p)\right] d \Gamma ; \quad P \in \Omega ; p, q \in \Gamma
\end{aligned}
$$

As integrais envolvem limites de domínio, com isso, precisa-se de duas malhas, uma de domínio (método dos elementos finitos) e outra nos limites (método dos elementos de contorno).

Ao desenvolver equações a partir dos valores nodais, tem-se:

$$
\begin{aligned}
A u_{\Omega} & =f B+C a \text { (no domínio) } \\
D u_{\Omega} & =E f+F a \text { (no contorno) } \\
A & =\int_{\Omega} \Psi(Q)^{T} \Psi(Q) d \Omega
\end{aligned}
$$




$$
\begin{gathered}
B=\int_{\Omega} \Psi(Q)^{T} G_{\Gamma}(Q) d \Omega \\
C=\int_{\Omega} \Psi(Q)^{T} G_{\Omega}(Q) d \Omega \\
D=\int_{\Gamma} \phi(q)^{T} \phi(q) d \Gamma \\
E=\int_{\Gamma} \phi(q)^{T} G_{\Gamma}(q) d \Gamma \\
F=\int_{\Gamma} \phi(q)^{T} G_{\Omega}(q) d \Gamma \\
G_{\Gamma}(Q)=\int_{\Gamma} G^{T}(p, Q) \phi(p) d \Gamma \\
G_{\Omega}(Q)=\int_{\Omega} G^{T}(P, Q) \Psi(P) d \Omega \\
G_{\Gamma}(Q)=\int_{\Gamma} G^{T}(p, q) \phi(p) d \Gamma \\
G_{\Omega}(Q)=\int_{\Omega} G^{T}(p, q) \phi(p) d \Gamma
\end{gathered}
$$

As matrizes $\Psi(Q)$ e $\phi(p)$ são as matrizes de forma no domínio e no contorno. Os pontos escritos de letra maiúscula são de domínio e de letra minúscula são de contorno.

$G_{\Gamma}(Q), G_{\Omega}(Q), G_{\Omega}(q) e G_{\Omega}(q)$ são as projeções das funções de Green sobre o contorno e o domínio que são mostradas nas Eq. (29), (30), (31) e (32).

\section{RESULTADOS}

Nesse trabalho, usou-se funções de forma quadrática para a construção de nove nós langrageana de elementos finitos e três nós de elementos de contorno.

Na Fig. 3 é mostrada a malha de elementos finitos e a malha de elementos de contornos.

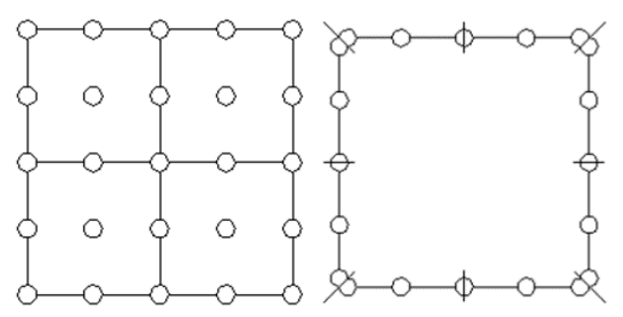

Figura 3. Malha de elementos finitos e Malha de elementos de contorno.

É usada a dupla simetria para discretizar o problema e as condições de contorno são "ditas" para o programa da maneira que é mostrada na Fig. 4. 


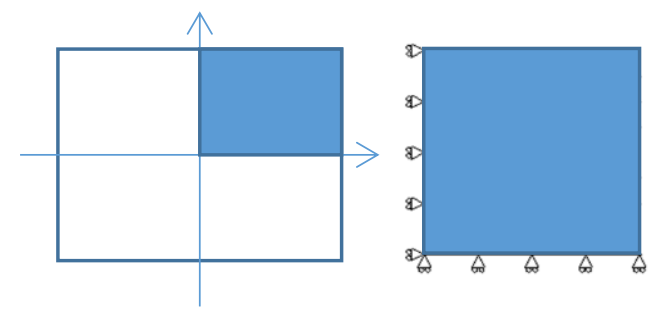

Figura 4. Discretização da Placa e Condições de contorno da placa discretizada com dupla simetria.

\subsection{Viga Laminada de Material Isotrópico}

O primeiro exemplo que foi processado é de uma viga (engastada e livre) formada por uma lâmina de material isotrópico e homogêneo, representada na Fig. 5. A viga foi modelada com elementos retangulares, variando o tamanho das malhas para análise de melhor convergência.

As propriedades mecânicas do material são: módulo de elasticidade $E=125 \mathrm{GPa}$ e coeficiente de Poisson $v=0,30$. As características geométricas são: comprimento $L=2 m$, largura da camada $b=0,05 m$ e espessura $h=0,001 \mathrm{~m}$. Na extremidade livre foi aplicada uma carga de $F=100 \mathrm{~N}$.

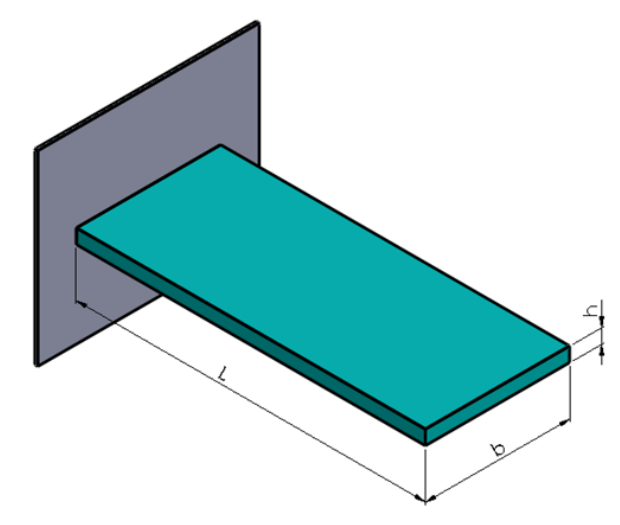

Figura 5. Viga piezoelétrica engastada-livre.

O deslocamento máximo foi encontrado de forma analítica pela expressão $\left(w_{\max }=-\frac{F L^{3}}{3 E I}\right)$.

A Tab. 2 mostra a comparação entre a solução analítica de $0,0512 m$ e a solução obtida nesse trabalho pelo MMFGL do deslocamento máximo $\left(w_{\max }\right)$ em metros $(m)$, com várias malhas $(L x b)$ e com o erro em relação à solução analítica de acordo com cada malha.

Tabela 2. Resultados obtidos de deslocamento máximo da viga de material isotrópico.

\begin{tabular}{|l|l|l|l|l|}
\hline Analítica & Malha 1x1 & Malha 2x2 & Malha 4x2 & Malha 8x2 \\
\hline 0,0512 & 0,0665 & 0,0503 & 0,0508 & 0,0510 \\
\hline Erro & $29,883 \%$ & $1,789 \%$ & $0,787 \%$ & $0,392 \%$ \\
\hline
\end{tabular}

No Fig. 6 tem-se a tensão máxima nas fibras comprimidas e tracionadas no engaste. A Tensão Máxima é de 1,491 $\mathrm{GPa}$, um erro de aproximadamente $4 \%$ em relação à tensão calculada analiticamente. 


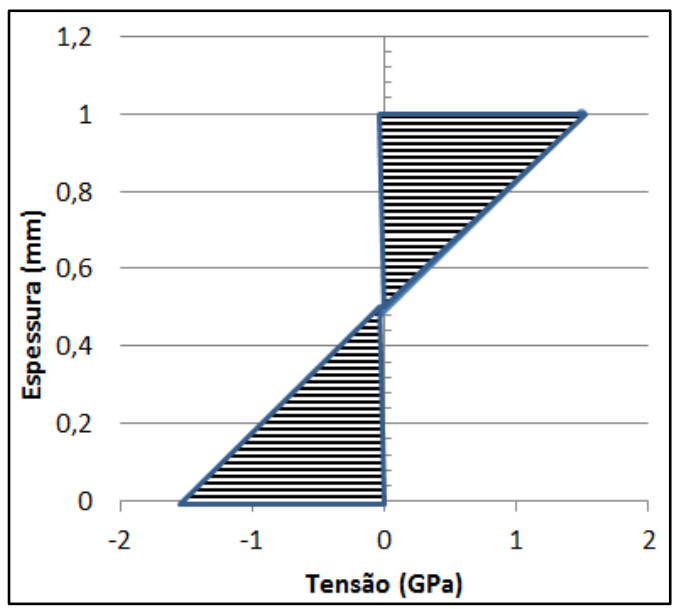

Figura 6. Tensões Mecânicas em $\boldsymbol{x}$ no Engaste da viga.

\subsection{Viga Laminada de Material Compósito Piezoelétrico (PVDF)}

Um exemplo clássico usado por muitos autores é o primeiro exemplo piezoelétrico que foi avaliado nesse trabalho. Trata-se da determinação do deslocamento máximo da viga engastada livre da Fig. 7 que é composta por duas camadas de polímero piezoelétrico quando é submetida a uma tensão elétrica.

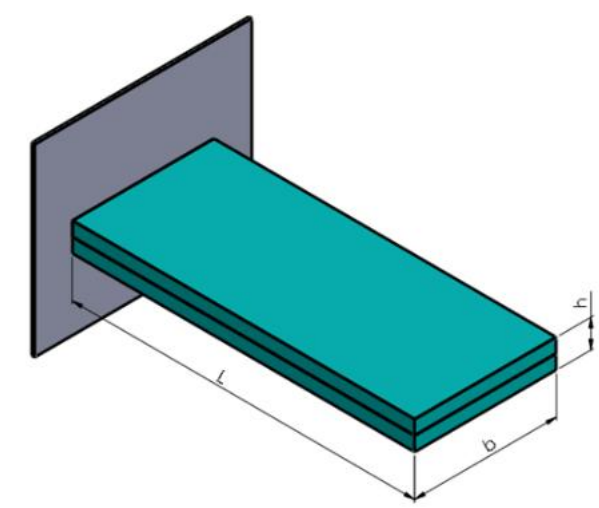

Figura 7. Viga piezoelétrica engastada livre.

Suas propriedades geométricas são: $L=0,10 \mathrm{~m}, h=0,001 \mathrm{~m}$ e $b=0,005 \mathrm{~m}$.

As propriedades mecânicas e elétricas do material são: $E=2.10^{9} \mathrm{~Pa}, \quad G=0,78.10^{9} \mathrm{~Pa}, \mathrm{v}=0,29, e=$ $0,046 C / \mathrm{m}^{2}$ e $\varepsilon=106,2 \cdot 10^{-12} \mathrm{~F} / \mathrm{m}$.

A viga foi modelada a partir de uma malha com elementos retangulares, foram testadas várias malhas.

Foi estudado o deslocamento sofrido quando é aplicada uma tensão elétrica de $1 V$ verticalmente no centro da extremidade livre.

A solução analítica do deslocamento $\left(w_{\max }\right)$ foi determinada segundo a Eq. (33), mostrada por Piefort (2001) para este caso.

$$
w_{\max }=-\frac{3}{2} \frac{d_{31} V}{h^{2}} L^{2}
$$

Sendo: $d_{31}=175 \cdot 10^{-12} \mathrm{~m} / \mathrm{V}$.

O resultado obtido por essa equação é de $3,45 \cdot 10^{-7} \mathrm{~m}$. A Tab. 3 mostra a comparação de várias malhas com a solução analítica, mostrando o erro percentual. Os resultados mostrados na tabela estão demonstrados com a malha $(L x b)$. 
Tabela 3. Resultados obtidos de deslocamento máximo da viga laminada de material piezoelétrico.

\begin{tabular}{|c|c|c|c|c|}
\hline Analítica & Malha 1x1 & Malha 2x2 & Malha 4x2 & Malha 8x2 \\
\hline $3,45.10^{-7}$ & $6,207.10^{-7}$ & $3,660.10^{-7}$ & $3,532.10^{-7}$ & $3,530.10^{-7}$ \\
\hline Erro & $79,913 \%$ & $6,089 \%$ & $2,377 \%$ & $2,319 \%$ \\
\hline
\end{tabular}

Os resultados do deslocamento máximo foram comparados a outros trabalhos e estão especificados na tabela. Conforme podem ser observados na Tab. 4 os resultados estão bem próximos dos outros autores. Os resultados foram retirados da tese de Isoldi (2008) e da dissertação de Torres (2008). Todos os resultados estão todos em metros $(m)$.

Tabela 4. Comparação dos resultados obtidos do deslocamento máximo.

\begin{tabular}{|l|c|}
\hline Isoldi (Malha 5 $x 1$ com 10 elementos triangulares) & $3,138.10^{-7}$ \\
\hline Lima Jr. (10 elementos de viga de Euler-Bernoulli) & $3,450.10^{-7}$ \\
\hline Lima Jr. (10 elementos de viga de Timoshenko) & $3,700.10^{-7}$ \\
\hline Lima Jr. (20 elementos de viga de Trilinear de 8 nós) & $3,340.10^{-7}$ \\
\hline Piefort & $3,300.10^{-7}$ \\
\hline Torres (Enriquecimento de $1^{\circ}$ grau com Malha 11x3) & $3,4512.10^{-7}$ \\
\hline Torres (Enriquecimento de $2^{\circ}$ grau com Malha 11x6) & $3,4514.10^{-7}$ \\
\hline Tzou (Numérica) & $3,300.10^{-7}$ \\
\hline Tzou (Analítica) & $3,450.10^{-7}$ \\
\hline Tzou (Experimento) & $3,150.10^{-7}$ \\
\hline Presente estudo & $\mathbf{3 , 5 3 0 . 1 0 ^ { - 7 }}$ \\
\hline
\end{tabular}

No Fig. 8, tem-se a tensão máxima (em $k P a$ ) nas fibras comprimidas e tracionadas no engaste. A Tensão Máxima é de 11,63 KPa, um erro de aproximadamente $8 \%$ em relação à tensão calculada analiticamente .

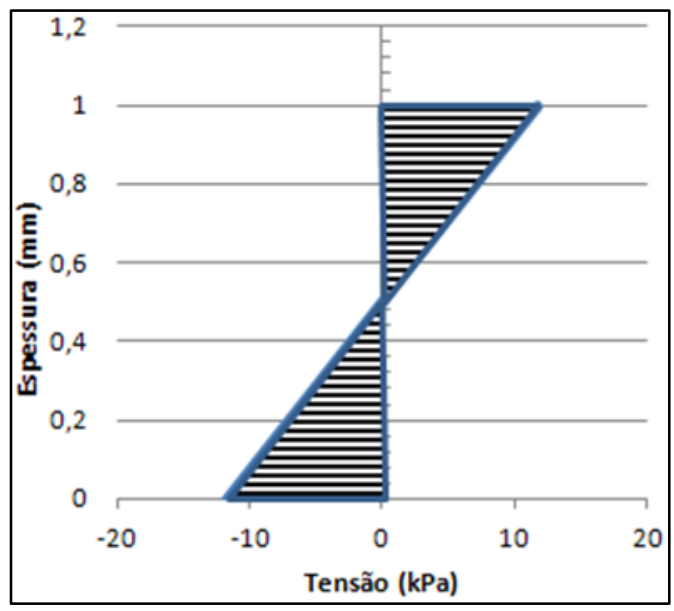

Figura 8. Tensões Mecânicas em $x$ no Engaste da viga.

\subsection{Viga Laminada de Material Compósito Piezoelétrico (PVDF) e com Material Isotrópico}

Nesse exemplo trabalha-se com duas lâminas de material isotrópico e uma lâmina de material piezoelétrico entre elas.

Esse caso foi deduzido analiticamente por Piefort (2001) para determinação do deslocamento máximo da viga engastada livre da Fig. 9 que é composta por duas camadas de material isotrópico (uma placa em cima e outra em baixo) e uma camada de material piezoelétrico.

As características geométricas e propriedades do material são idênticas as do exemplo anterior. Só um detalhe para a espessura $\mathrm{h}=0,001 \mathrm{~m}$ que é somente da lamina piezoelétrico e as outras duas laminas também tem $0,001 \mathrm{~m}$ de espessura.

É aplicada uma tensão de $1 \mathrm{~V}$ verticalmente no centro da extremidade livre da camada piezoelétrico. 


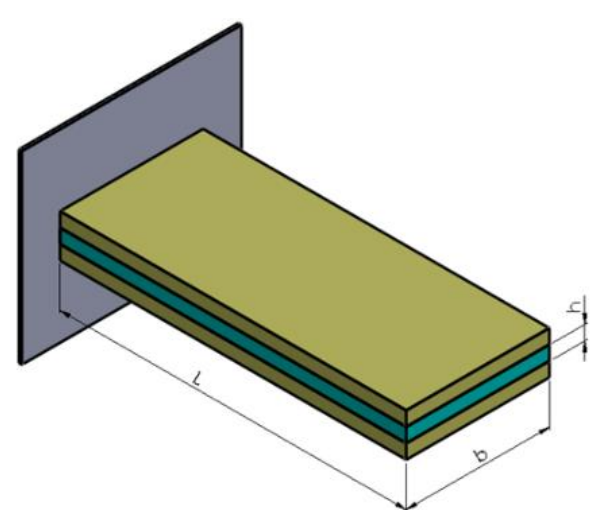

Figura 9. Viga engastada livre com uma camada piezoelétrico.

A solução analítica foi determinada segundo a Eq. (34), mostrada por Piefort (2001) para este caso.

$$
w=L d_{15} \frac{V}{h}
$$

Sendo: $d_{15}=515 \cdot 10^{-12} \mathrm{~m} / \mathrm{V}$.

Resolvendo por essa solução analítica o deslocamento máximo é de 5,156.10 $10^{-8}$.

A Tab. 5 mostra o deslocamento máximo pelo método analítico, os deslocamentos máximos obtidos pelo MMFGL de acordo com cada malha e indica o erro em relação à solução analítica.

Tabela 5. Resultados obtidos de deslocamento máximo da viga laminada com camada de material piezoelétrico.

\begin{tabular}{|c|c|c|c|c|}
\hline Analítica & Malha 1x1 & Malha 3x1 & Malha 3x3 & Malha 5x3 \\
\hline $5,156.10^{-8}$ & $8,189.10^{-8}$ & $4,934 \cdot 10^{-8}$ & $5,337 \cdot 10^{-8}$ & $5,194 \cdot 10^{-8}$ \\
\hline Erro & $58,82 \%$ & $4,49 \%$ & $3,51 \%$ & $0,73 \%$ \\
\hline
\end{tabular}

No Fig. 10, tem-se a tensão máxima (em $k P a$ ) nas fibras comprimidas e tracionadas no engaste da lâmina piezoelétrico. A Tensão Máxima é de 4,91 KPa, um erro de aproximadamente 1,5\% em relação à tensão calculada analiticamente.

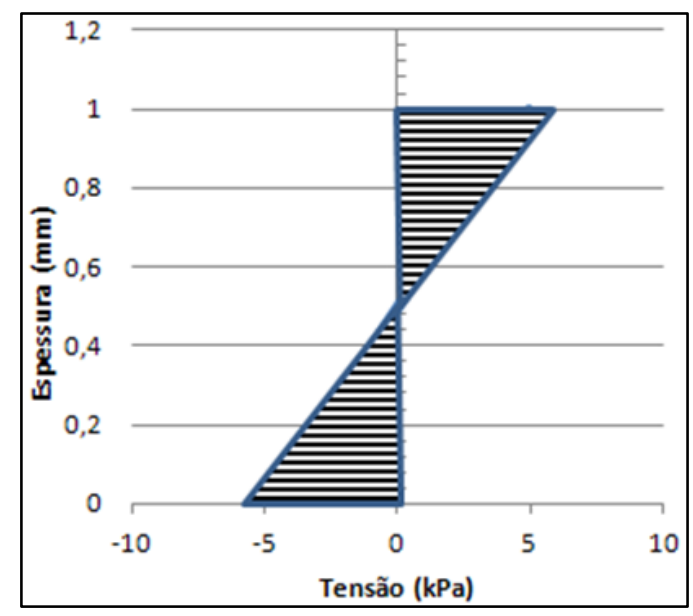

Figura 10. Tensões Mecânicas em $x$ no Engaste da viga.

\section{CONCLUSÃO}

Neste trabalho foi realizada uma modelagem computacional usando a formulação e fazendo a implementação do Método Modificado da Função de Green Local (MMFGL) para análise estática linear de placa laminada com material compósito piezoelétrico com tensões elétricas aplicadas, possibilitando alterar os tipos e quantidades de lâminas. 
Primeiramente, foi feito um levantamento teórico de conceitos de piezoeletricidade, materiais compostos, material compósito piezoelétrico, placas laminadas, teorias sobre placas, Teoria de Deformação de Cisalhamento de Primeira Ordem (FSDT) e do Método Modificado da Função de Green Local (MMFGL).

O Método Modificado da Função de Green Local tinha sido usado em problema de Placa de Material Compósito. Neste trabalho, com base no que já tinha sido produzido, foram implementadas rotinas com a introdução dessa estrutura inteligente, que é o efeito piezoelétrico. Foi implementado em ambiente FORTRAN.

$\mathrm{O}$ acoplamento eletromecânico foi representado através de formulação matemática, considerando as propriedades mecânicas e elétricas do material. O modelo foi validado através de comparações de resultados de deslocamento e tensões mecânicas com soluções analíticas e soluções encontradas na literatura.

Foi trabalho com várias malhas, desde uma malha grosseira, até uma malha que tivesse uma convergência para a resposta esperada.

Os exemplos rodados mostram um bom desempenho do Método Modificado da Função de Green Local em problemas mecânicos e eletromecânicos.

Um detalhe que foi notado também, é que mesmo com malhas grosseiras o método gera boas respostas, por conseguir calcular os fluxos de contorno com boa precisão.

Além de determinar deslocamentos, o método apresenta uma alternativa para determinação de tensões mecânicas.

\section{REFERÊNCIAS}

DELla GIUSTINA, E. 2013. Análise de Material Compósito Piezoelétrico pelo MMFGL, dissertação de mestrado. Programa de Pós Graduação em Engenharia Mecânica, Pontifícia Universidade Católica do Paraná.

FARIA, A. W., 2006. Modelagem por elementos finitos de placas compostas dotadas de sensores e atuadores piezoelétricos: implementação computacional e avaliação numérica, dissertação de mestrado, Faculdade de Engenharia Mecânica da UFU.

GODOY, T. C., 2008. Modelagem de placas laminadas com materiais piezoelétricos conectadas à circuitos shunt resistivo-indutivo, dissertação de mestrado, Escola de Engenharia de São Carlos da USP.

ISOLDI, L. A., 2008. Análise estática e dinâmica de estruturas delgadas de materiais compostos laminados incluindo materiais piezelétricos, tese de doutorado, Universidade Federal do Rio Grande do Sul.

MACHADO, R. D., 1992. Desenvolvimento do Método Modificado da Função de Green Local para a Solução de Placas Laminadas de Materiais Compostos, tese de doutorado, Florianópolis - SC - Brasil, Universidade Federal de Santa Catarina.

MACHADO, R. D.; BARBIERI, R.; SILVA, M. P.; TASSINI, A.; DELlA GIUSTINA, E., 2013. The MMFGL applied to Laminated Composite Plates, Proceedings of the XXXIV Ibero Latin American Congress on Computational Methods in Engineering (CILAMCE). Pirenopólis, GO, Brazil.

MACHADO, R. D.; ABDALLA FILHO, J. E., SILVA, M. P., 2007. Stiffness loss of laminated composite plates with distributed damage by the modified local Green's function method, Proceedings of the Composite Structures, Programa de Pós Graduação em Engenharia Mecânica, Pontifícia Universidade Católica do Paraná.

PIEFORT, V., 2001. Finite Element Modelling of Piezoelectric Active Structures, tese de doutorado, Universidade Livre de Bruxelas.

TORRES, D. A. F., 2008. Método dos Elementos Finitos Generalizados aplicado à análise de placas laminadas compostas inteligentes, dissertação de mestrado, Universidade Federal de Santa Catarina.

\section{RESPONSABILIDADE AUTORAL}

“O(s) autor(es) é(são) o(s) único(s) responsável(is) pelo conteúdo deste trabalho”. 


\title{
STATIC ANALYSIS OF LINEAR BEAM OF LAMINATED PVDF
}

\author{
Edgar Della Giustina, edgar.giustina @pr.senai.br ${ }^{1}$
}

${ }^{1}$ Faculty of Technology SENAI CIC - Department of Education... Rua Senador Accioly Filho, 298, Cidade Industrial, CEP 81310-000, Curitiba, PR - Brasil

I

Abstract. The present work analyzes the behavior of piezoelectric composite beams (PVDF). The analysis occurs by developing routines in FORTRAN compiler to include properties and constitutive matrices of piezoelectric materials. For the resolution used the Theory of First Order Shear Deformation (FSDT). The numerical method used was the Modified Method of Green's function Local (MMFGL). To obtain best results in different sized meshes were created to achieve a convergence of results. The results obtained were compared with various numerical methods in the literature and analytical solutions of piezoelectric materials subjected to electrical voltages. Piezoelectric materials can be used in structures that need good flexibility and small displacements. Thus, this article aims to show a collaboration to better understand the behavior of the characteristics of piezoelectric materials compared with those without such properties.

Keywords: Piezoelectricity, Composite Material, Method of Modified Local Green's function. 